\title{
Structural but not functional neuroplasticity one year after effective cognitive behaviour therapy for social anxiety disorder
}

Kristoffer N.T. Månsson, Alireza Salami, Per Carlbring, C.-J Boraxbekk, Gerhard Andersson and Tomas Furmark

\section{Journal Article}

\section{Tweet}

N.B.: When citing this work, cite the original article.

Original Publication:

Kristoffer N.T. Månsson, Alireza Salami, Per Carlbring, C. -J Boraxbekk, Gerhard Andersson and Tomas Furmark, Structural but not functional neuroplasticity one year after effective cognitive behaviour therapy for social anxiety disorder, Behavioural Brain Research, 2017. 318, pp.45-51.

http://dx.doi.org/10.1016/j.bbr.2016.11.018

Copyright: Elsevier

http://www.elsevier.com/

Postprint available at: Linköping University Electronic Press

http://urn.kb.se/resolve?urn=urn:nbn:se:liu:diva-132638






\section{Structural but not functional neuroplasticity one year after effective cognitive behaviour therapy for social anxiety disorder}

Kristoffer N.T. Månsson ${ }^{\mathrm{a}, \mathrm{b}^{*}}$

Alireza Salami ${ }^{\mathrm{c}, \mathrm{d}}$

Per Carlbringe

C-J Boraxbekk $k^{\mathrm{d}, \mathrm{f}, \mathrm{g}}$

Gerhard Andersson ${ }^{\mathrm{a}, \mathrm{b}}$

Tomas Furmark ${ }^{\mathrm{h}}$

a Department of Behavioural Sciences and Learning, Psychology, Linköping University, Linköping, Sweden

${ }^{\mathrm{b}}$ Department of Clinical Neuroscience, Karolinska Institutet, Stockholm, Sweden



${ }^{\mathrm{d}}$ Umeå Centre for Functional Brain Imaging (UFBI), Umeå University, Umeå, Sweden

${ }^{\text {e }}$ Department of Psychology, Stockholm University, Stockholm, Sweden

${ }^{\mathrm{f}}$ Centre Demographic and Aging Research (CEDAR), Umeå University, Umeå, Sweden

${ }^{g}$ Danish Research Centre for Magnetic Resonance, Centre for Functional and Diagnostic

Imaging and Research, Copenhagen University Hospital Hvidovre, Denmark

${ }^{\mathrm{h}}$ Department of Psychology, Uppsala University, Uppsala, Sweden

*Address for correspondence: Linköping University, Department of Behavioural Sciences and Learning, Psychology, SE-581 83 Linköping, Sweden. Tel: +46 (0)70 5085082; Fax: +46

(0)13 282145, E-mail address: kristoffer.nt.mansson@liu.se (K.N.T. Månsson). 


\begin{abstract}
Effective psychiatric treatments ameliorate excessive anxiety and induce neuroplasticity immediately after the intervention, indicating that emotional components in the human brain are rapidly adaptable. Still, the interplay between structural and functional neuroplasticity is poorly understood, and studies of treatment-induced long-term neuroplasticity are rare. Functional and structural magnetic resonance imaging (using 3 Tesla MRI) was performed in 13 subjects with social anxiety disorder on 3 occasions over 1 year. All subjects underwent 9 weeks of Internet-delivered cognitive behaviour therapy in a randomized cross-over design and independent assessors used the Clinically Global Impression-Improvement (CGI-I) scale to determine treatment response. Gray matter (GM) volume, assessed with voxel-based morphometry, and functional blood-oxygen level-dependent (BOLD) responsivity to selfreferential criticism were compared between treatment responders and non-responders using 2 $\times 2$ (group $\times$ time; pretreatment to follow-up) ANOVA. At 1-year follow-up, 7 (54\%) subjects were classified as CGI-I responders. Left amygdala GM volume was more reduced in responders relative to non-responders from pretreatment to 1-year follow-up $(Z=3.67$, Family-Wise Error corrected $p=0.02)$. In contrast to previous short-term effects, altered BOLD activations to self-referential criticism did not separate responder groups at follow-up. The structure and function of the amygdala changes immediately after effective psychological treatment of social anxiety disorder, but only reduced amygdala GM volume, and not functional activity, is associated with clinical response 1 year after CBT.
\end{abstract}

Keywords: Amygdala; Neuroplasticity; Long-term; Social Anxiety Disorder; Cognitive Behavior Therapy 


\section{Introduction}

The human brain is rapidly adaptable. As detected by non-invasive, high-resolution magnetic resonance imaging (MRI), structural change in gray matter (GM) volume in the human brain may occur in less than a week by repetitive stimulation [1] and there is a growing body of studies showing short-term structural brain alterations after activities and interventions [2, 3]. Longitudinal intervention studies with GM outcomes are rare although neuroimaging studies of brain plasticity over the long-term may open new avenues to understanding and treatment of mental disorders.

Cognitive behaviour therapy (CBT) is an evidence-based treatment for a range of mental illnesses. To date, there are numerous imaging studies demonstrating functional changes in neural responsivity after CBT for social anxiety disorder [4-6], panic disorder [7], and depression [8]. In addition to this, we recently showed that the amygdala GM volume declined after 9 weeks of Internet-delivered CBT for social anxiety disorder, and that this structural change correlated with symptom improvement at the behavioural level [9]. Moreover, the amygdala GM volume reduction after treatment correlated with decreased amygdala blood-oxygen level-dependent (BOLD-fMRI) responsivity to a disorder-relevant fMRI task, i.e., self-referential criticism [9]. This result is in line with the basic notion that changes in neural activity, at least in part, are accounted for by changes in GM volume [10, 11]. While there are some multi-modal neuroimaging studies incorporating analyses of structure-function relationships, in psychotic disorders for instance [12], such studies are surprisingly rare in the psychiatric literature. Furthermore, long-lasting change of the structure-function interplay, has been an overlooked research question in psychiatric disorders. Only taking structural neuroplasticity into account, there are a few studies demonstrating long-term morphological changes after CBT, e.g., in obsessive-compulsive 
disorder [13] and spider-phobia [14], providing tentative evidence that effective psychological treatments can have long-lasting effects on brain GM volume. However, a crucial question is how long-term structural changes of this kind are affected by, or affects, neural activity.

The present study examined long-term effects of Internet-delivered CBT both on structural and functional neuroplasticity 1 year after treatment of social anxiety disorder. In line with the immediate effects at posttreatment [9], we expected to find similar long-term neuroplasticity, i.e., reduced amygdala GM volume along with diminished anxiety-related amygdala neural responsivity after successful treatment. Also, in accordance with our previous findings, we expected a correlation between reduced amygdala GM volume and symptom improvement at 1-year follow-up.

\section{Methods}

\subsection{Subjects}

The subjects were recruited from a previously randomized controlled trial of CBT for social anxiety disorder $[9,15,16]$. Twenty-one subjects were invited via e-mail to participate in a 1year follow-up assessment. All accepted to answer self-reported questionnaires and to partake in a clinical interview via telephone. Thirteen subjects (59\%, 13/22) accepted to undergo an additional MRI, and were thus included in the current study. We found no demographic or clinical pretreatment differences (all $p^{\prime}$ s $>0.23$ ) between subjects who did vs. did not participate in the 1-year follow-up MRI session. Demographic and clinical characteristics of the 13 participants are shown in Table 1 . Besides a trend $(p=0.06)$ for lower education level in long-term non-responders (vs. responders), there were no significant differences at pretreatment between the responder subgroups (all $p^{\prime}$ s $>0.37$ ). Prior to treatment, all subjects suffered from social anxiety disorder according to DSM-IV [17]. One subject (classified as a 
non-responder at 1-year follow-up) had increased the sertraline dose from 50 to $100 \mathrm{mg}$ from posttreatment to 1-year follow-up. Those who accepted to participate in the long-term followup gave written informed consent and were reimbursed with about $€ 70$. The local ethical committee approved the study, which was pre-registered at ClinicalTrials.gov (ID: NCT01312571).

\subsection{Treatment}

As previously described $[15,16]$, the subjects were sequentially offered Internet-delivered CBT and attention bias modification (ABM) in a cross-over design and the subjects were randomized to start either with Internet-delivered CBT or ABM [15]. The therapist-guided Internet-delivered CBT protocol encompassed a 9-week text-based intervention with weekly online support from a psychologist. In line with previous studies, ABM was delivered for 4 weeks. In accordance with several randomized controlled trials [RCTs; 18, 19-21] Internetdelivered CBT was found to be effective while ABM performed less well [15].

\subsection{Assessment of clinical response}

As for the posttreatment assessments, clinical response at 1-year follow-up was determined using a clinical interview and the Clinically Global Impression-Improvement (CGI-I) scale [22]. The scale was administrated by 2 independent clinicians blind to the experimental conditions and prior evaluations. Participants with CGI-I scores indicating much or very much improvement were defined as treatment responders. In addition, the subjects responded to the gold standard Liebowitz Social Anxiety Scale (Self-report version; LSAS-SR) [23].

The subjects were also instructed to perform a 2-minute public speaking task after the MRI session, but, in contrast to the previous assessments [9], they did not have to carry out the task 
at 1-year follow-up. Prior to the expected speech task, anticipatory speech anxiety was assessed using a subjective unit of distress (SUDs) scale, i.e., the mean of fear and distress, rated separately on a min-max scale from 0-100. SUD ratings were completed at 4 time-points (approximately 60, 40, 30 and 1 min before the speech) while the subject was lying in the MRI scanner.

\subsection{BOLD-fMRI experimental task}

Stimuli were presented using the E-Prime 2.0 software (Psychology Software Tools, Pittsburgh, PA, USA), projected on a screen and viewed through a tilted mirror attached to the head coil. The experimental task has been described in detail elsewhere [9, 16, 24]. Briefly, we evaluated BOLD response to sentences expressing self-referential criticism (e.g., "You are stupid”; relative to criticism targeting a peer, e.g., "He is stupid”), presented for a maximum of 2500 ms. Subjects were instructed to read the sentences and then confirm this by pressing a button with the right hand. In addition, 96 fixation crosses (“+”) were randomly interspersed between the sentences and were also presented for 2500 ms. Each sentence and fixation cross was separated by a cross or circle presented for $500 \mathrm{~ms}$.

\subsection{Imaging}

\subsubsection{Structural and functional image acquisition}

All neuroimaging was performed in a 3T scanner (General Electric, Madison, WI, USA) equipped with a 32-channel head coil. The structural T1-weighted images were acquired with a voxel size of $0.5 \times 0.5 \times 1 \mathrm{~mm}^{3}$, including 180 slices per subject and a field of view of 250 mm. The following parameters were used for the EPI sequence: TR: $2000 \mathrm{~ms}$, TE: $30 \mathrm{ms,}$ flip-angle: $80^{\circ}$, FOV: $250 \times 250 \mathrm{~mm}^{2}$, matrix size: $96 \times 96$, in-plane resolution: $2.6 \times 2.6 \mathrm{~mm}$, 
and each volume contained 37 slices (3.4 mm thick). Before the image acquisition, ten dummy scans were collected to allow for equilibration of the fMRI signal.

The Statistical Parametric Mapping Software version 8 (SPM8; Wellcome Department of Cognitive Neurology, London, UK) and the MATLAB (Mathworks Incorporation, Natick, MA, USA) were used to perform neuroimaging analyses. The T1-weighted images were preprocessed using the VBM8 toolbox (Christian Gaser, University of Jena, Department of Psychiatry) that calculated modulated normalized GM volumes and allowed for comparing the amount of tissue corrected for individual brain sizes. Default settings were used, i.e., bias correction for signal inhomogeneities, and spatially normalized with diffeomorphic anatomical registration through exponentiated lie algebra (DARTEL). To identify outliers, quality control was carried out using the sample homogeneity test, and we found that covariance was within 2 standard deviations, thus, no outliers were excluded. The VBM8 preprocessing included longitudinal MRI assessments corresponding to a) pretreatment vs. 1year follow-up, and b) posttreatment vs. 1-year follow-up. Structural scans were segmented into gray and white matter, and the GM volumes were non-linearly normalized to the Montreal Neurological Institute (MNI) template, modulated, and smoothed using an $8 \mathrm{~mm}$ full-width half-maximum (FWHM) isotropic Gaussian kernel.

Functional MRI data were first rigidly aligned to the middle image volume of each run to correct for head movements. The realigned images were then corrected for acquisition time differences between slices within each volume. A within-subject rigid registration was conducted to align functional and structural images together. 
We co-registered the functional scans to a longitudinal mean structural image. Functional scans were subsequently warped to MNI152 standard space (using the transformation parameters that normalized GM images into the MNI space) and smoothed with an $6 \mathrm{~mm}$ fullwidth half-maximum (FWHM) isotropic Gaussian kernel. Thus, both fMRI and VBM images were in the same space and had the same voxel size (i.e., $1.5 \times 1.5 \times 1.5 \mathrm{~mm}^{3}$ ).

For the BOLD-fMRI paradigm, subject-specific contrasts (self-referential criticism vs. otherreferential criticism) were generated with voxel-wise general linear models (GLM). Each condition was modeled as a box-car function, convolved with the hemodynamic response function and filtered using a $128 \mathrm{~s}$ high-pass filter. In addition, 6 motion parameters derived from the realignment algorithm were included in the model to account for motion artifacts.

\subsubsection{Data analysis}

The randomized cross-over study design prevented us from evaluating the trajectories from pre to post to 1-year follow-up (i.e., 3 time points) in all 13 subjects because only half of the participants had received CBT at post-treatment. Hereafter, the cross-over ensured that all subjects did receive CBT before the 1year follow-up assessment. Long-term treatment effects (GM volume, and BOLD-fMRI to self-referential criticism) were evaluated using flexible factorial design (ANOVA $2 \times 2$ ) in SPM. In order to assess long-term effects we implemented group (long-term CBT responders as compared to non-responders) over time (pretreatment to 1 year follow-up). Changes from posttreatment to follow-up were evaluated in the subset of patients $(n=7)$ initially treated with CBT in the cross-over design. Within-group changes (i.e., pre- or posttreatment to 1-year) were evaluated in paired $t$-test's. Brain-behaviour correlations were performed with linear regression implementing changed beta weights and 
altered anxiety symptoms from pre-, or posttreatment to 1-year follow-up. Anticipatory speech anxiety corresponded to the final rating of fear and distress, i.e., assessed right before the expected speech task.

In accordance with our previous study, the left and right amygdala were regions of interest (ROIs) as defined by the Wake Forest University AAL PickAtlas [25]. The threshold level was set at $p<0.05$ with a Family-Wise Error (FWE) on voxel-wise comparisons in small volume corrections.

Cross-sectional demographic and clinical variables were compared using $t$-test's or Fisher's exact test, longitudinal clinical variables were analyzed with generalized estimating equation with an exchangeable correlation structure.

\section{Results}

\subsection{Treatment outcome}

Of the 13 participants in the fMRI assessment at 1-year follow-up, 7 were classified as treatment responders (54\%) and 6 as non-responders (46\%) according to the CGI-I. Anticipatory social anxiety (i.e., SUDs) decreased from pretreatment (Mean/StD = 42/26) to 1-year follow-up (Mean/StD = 30/24; $\beta=-6.2, Z=-2.98, p<0.05)$, see also Figure 1 . Similarly, LSAS-SR scores decreased from pretreatment $($ Mean/StD $=74.00 / 15.5)$ to 1-year follow-up (Mean/StD = 48.38/23.6; $\beta=-25.6, Z=-6.81, p<0.001)$. CGI-I responder subgroups could not be differentiated on SUDs or LSAS-SR alterations (all p’s > 0.16). 


\subsection{Structural and functional neuroplasticity associated with long-term treatment response}

After controlling for total intracranial brain volume, age, sex, and concurrent SSRI

medication, we found a significant (group $\times$ time) interaction effect such that treatment responders, relative to non-responders, had significantly greater reduction in left amygdala GM volume from pretreatment to 1-year follow-up (Table 2 and Figure 2-3). GM loss in the right amygdala was not significant after FWE correction, see Table 2. As shown in Figure 2, there were no outliers affecting this result. Moreover, pre-treatment GM volume of the left and right amygdala did not separate long-term responders from non-responders $(Z<2.32$, $p \mathrm{FWE}>0.471)$.

In contrast to our previous study on short-term effects,[9] we did not find a similar interaction (group $\times$ time) effect for BOLD-responsivity to self-referential criticism at the 1-year followup (all p’s>0.05).

\subsection{Association between changes in anxiety symptoms and amygdala gray matter volume}

In accordance with our previous finding [9], reduced state-related anticipatory anxiety from pretreatment to follow-up was positively associated with reduced left amygdala $(Z=3.17$, $p \mathrm{FWE}=0.05, \mathrm{xyz}[-26,-4,-23] k=104)$, but not right amygdala $(Z=2.66, p \mathrm{FWE}=0.17$, xyz[24,-3,-21], $k=70$ ) GM volume, see also Figure 4. No significant associations were noted between changes in amygdala GM and LSAS-SR.

\subsection{Neuroplastic changes from posttreatment to 1-year follow-up}

In subjects initially treated with CBT in the cross-over design $(n=7)$, long-term follow-up analyses showed that GM volume declined from post CBT to 1-year follow-up in the right amygdala $(Z=4.12, p F W E=0.01, x y z[22,-7,-14], k=222)$, but not significantly after FWE 
correction in the left amygdala $(Z=2.95, p F W E=0.18$, xyz[-16,-1,-15], $k=161$ ), see Figure 5. Over the same time-period, attenuated anticipatory speech anxiety correlated with reduced amygdala GM volume in the right $(Z=3.62, p F W E=0.05, x y z[20,3,-15], k=44)$, but not in the left amygdala $(Z=2.94 p F W E=0.16, x y z[-26,2,-17], k=32)$.

Moreover, when including the other cross-over treatment arm in the analysis, i.e., also the 6 subjects initially treated with ABM receiving CBT 2 months later, amygdala GM volume declined significantly both in the left $(Z=4.13, p F W E<0.05$, xyz[-18,-1,-15], $k=219)$ and right amygdala $(Z=4.72, p \mathrm{FWE}<0.05$, $\mathrm{xyz}[24,-9,-14], k=245)$ from posttreatment to 1 year follow-up. There were no significant changes in fMRI BOLD-responsivity from postCBT to follow-up.

\section{Discussion}

Following our previous report on neuroplasticity immediately after CBT for social anxiety disorder [9] we expected to find persistent treatment effects also at 1-year follow-up with treatment-related reduction of amygdala GM volume, as well as decreased BOLDresponsivity. There was only partial support for our prediction, i.e., decreased amygdala GM volume from pretreatment to 1-year follow-up was evident in responders but decreased amygdala neural responsivity did not discriminate between long-term treatment responders and non-responders over the same time period. Nonetheless, the reduced amygdala GM volume previously seen from pre- to posttreatment [9] was also detected in responders 1 year after treatment, and our additional analyses suggested that there was further GM reduction between posttreatment and 1-year follow-up correlating with decreased state-related anxiety over the same period. 
There is growing evidence that anxiolytic treatments alter neural responsivity over shorter time periods while only a small number of studies have investigated long-term brain alterations [13, 14]. However, similar to the current study, Schienle and colleagues [14] found suggestive evidence for decreased amygdala GM volume 6 months after CBT for spider phobia.

The current study adds to the notion that effective psychological treatments impact human neuroplasticity, both in the short- and long-term $[4,5,7,9,13]$. While we found that amygdala BOLD responsivity was attenuated at short-term, but not at long-term follow-up, this did not correspond to a different clinical outcome, suggesting that decreased anxiety after treatment cannot solely be explained by attenuated amygdala neural activation. It is possible that the treatment effect on neural responsivity alters over time. For instance, amygdala response diminishes immediately after the treatment, but over the long-term there may be other regions becoming more operative. In a series of experiments, Goldin and colleagues [26] have shown that individuals with social anxiety disorder, relative to healthy controls, require more time to recruit emotion regulatory prefrontal regions, and interestingly they also demonstrated that these patients became faster to recruit dorsolateral and dorsomedial prefrontal regions immediately after effective CBT [4]. One reason as to why we did not find persistent group differences in amygdala neural responsivity at the 1-year follow-up could be that amygdala BOLD response returns to baseline over a longer period of time, which may be in contrast to the treatment effect on prefrontal regions involved in emotion regulation. However, this needs to be further studied. Furthermore, Wenger and colleagues [27] recently showed that training-induced motor cortex neuroplasticity in healthy individuals is inverselyquadratic over time, suggesting that rapid increases in GM volume after initial training returns to baseline in the long-term, even though the behavioural effect persists. Interestingly, the 
structural neuroplasticity occurred without any significant change in functional responsivity within the same brain region [27]. In comparison, we found GM reduction to be stable over time while altered neural responsivity returned to baseline. Longitudinal multi-modal neuroimaging studies with multiple assessments over longer periods of time are needed to further uncover the complex nature of neuroplasticity [27].

While the multi-modal approach and the longitudinal design are strengths of the current study, it is limited by low statistical power as only 13 subjects participated in the 1-year follow-up. However, the structural long-term changes in the amygdala were in line with our previous study on short-term effects [9], and no apparent outliers were noted (see Figure 2). At 1-year follow-up, the subjects had sequentially received 2 Internet-delivered interventions, i.e., CBT and ABM. According to a previous randomized clinical study [28] there was no additive effect of ABM to CBT, but this cannot completely be ruled out in the current trial. Immediately after treatment, somewhat opposing effects were noted of the 2 interventions on amygdala GM volume and neural responsivity (see Månsson et al., 2016 [9]). Briefly, we found that the amygdala GM volume decreased after CBT, and that the effect after ABM was leaning towards the opposite direction. This may be another reason as to why we did not find any significant between-group differences from pretreatment to long-term follow-up on BOLD-fMRI amygdala responsivity, while there were differences in GM volume. Another possible explanation is related to test-retest reliability. Cannon et al., showed a good intraclass correlation (0.85) for GM volume in the amygdala, as determined by VBM [29] (same technique used herein). However, there is no such reliability assessment on the BOLD-fMRI experiment used in the current study. 


\section{Conclusions}

Despite these limitations, our data suggest that the amygdala changes immediately after effective psychological interventions for social anxiety disorder but only structural, and not functional, changes are associated with long-term clinical response to CBT. There was, however, suggestive evidence that additional amygdala GM reduction from posttreatment to 1-year follow-up correlated with attenuated state-related anxiety over the same period. In general, the current study replicated our previous findings on structural neuroplasticity, suggesting that changes in brain morphometry occurring immediately after CBT also are valid in a year-long perspective. 
Acknowledgements: Ann-Katrine Larsson and the staff of the Umeå Functional Brain Imaging Centre (UFBI) providing excellent research conditions. Carl-Johan Uckelstam contributed as a therapist in the cross-over treatment group. Funding had no role in the study setup, data interpretation, or reporting. This study was supported by grants from Swedish Research Council (Dr. Furmark), Linköping University (Dr. Andersson), Swedish Council for Working Life and Social Research (Dr. Carlbring, and Dr. Furmark), Capio Research Foundation and PRIMA Research Foundation (MSc Månsson).

Competing interests: None declared.

Contributions: KM, CJB, PC, GA, and TF designed the study. KM, TF and AS contributed to the data analysis. KM drafted the first version of the manuscript. TF, CJB, AS, GA and PC were involved in the manuscript preparation and all authors read and approved the manuscript. 


\section{References}

[1] A. May, G. Hajak, S. Ganssbauer, T. Steffens, B. Langguth, T. Kleinjung, P. Eichhammer, Structural brain alterations following 5 days of intervention: dynamic aspects of neuroplasticity, Cereb Cortex 17(1) (2007) 205-10.

[2] S. Kühn, T. Gleich, R.C. Lorenz, U. Lindenberger, J. Gallinat, Playing Super Mario induces structural brain plasticity: gray matter changes resulting from training with a commercial video game., Mol Psychiatry 19(2) (2014) 265-271.

[3] M. Allen, M. Dietz, K.S. Blair, M. van Beek, G. Rees, P. Vestergaard-Poulsen, A. Lutz, A. Roepstorff, Cognitive-affective neural plasticity following active-controlled mindfulness intervention, J Neurosci 32(44) (2012) 15601-10.

[4] P.R. Goldin, M. Ziv, H. Jazaieri, K. Hahn, R. Heimberg, J.J. Gross, Impact of cognitive behavioral therapy for social anxiety disorder on the neural dynamics of cognitive reappraisal of negative self-beliefs: randomized clinical trial, JAMA Psychiatry 70(10) (2013) 1048-56.

[5] T. Furmark, M. Tillfors, I. Marteinsdottir, H. Fischer, A. Pissiota, B. Langstrom, M. Fredrikson, Common changes in cerebral blood flow in patients with social phobia treated with citalopram or cognitive-behavioral therapy, Arch Gen Psychiatry 59(5) (2002) 425-33.

[6] H. Klumpp, D.A. Fitzgerald, M. Angstadt, D. Post, K.L. Phan, Neural response during attentional control and emotion processing predicts improvement after cognitive behavioral therapy in generalized social anxiety disorder., Psychol Med (2014) 1-13.

[7] U. Lueken, B. Straube, C. Konrad, H.U. Wittchen, A. Strohle, A. Wittmann, B. Pfleiderer, C. Uhlmann, V. Arolt, A. Jansen, T. Kircher, Neural substrates of treatment response to cognitive-behavioral therapy in panic disorder with agoraphobia, Am J Psychiatry 170(11) (2013) 1345-55.

[8] C.H. Fu, S.C. Williams, A.J. Cleare, J. Scott, M.T. Mitterschiffthaler, N.D. Walsh, C. Donaldson, J. Suckling, C. Andrew, H. Steiner, R.M. Murray, Neural responses to sad facial expressions in major depression following cognitive behavioral therapy, Biol Psychiatry 64(6) (2008) 505-12.

[9] K. Månsson, A. Salami, A. Frick, P. Carlbring, G. Andersson, T. Furmark, C.J. Boraxbekk, Neuroplasticity in response to cognitive behavior therapy for social anxiety disorder, Transl Psychiatry 6 (2016) e727.

[10] L. Nyberg, A. Salami, M. Andersson, J. Eriksson, G. Kalpouzos, K. Kauppi, J. Lind, S. Pudas, J. Persson, L.-G. Nilsson, Longitudinal evidence for diminished frontal cortex function in aging, PNAS 107(52) (2010) 22682-22686.

[11] A. Salami, J. Eriksson, L. Nyberg, Opposing effects of aging on large-scale brain systems for memory encoding and cognitive control, J Neurosci 32(31) (2012) 10749-57.

[12] J. Radua, S. Borgwardt, A. Crescini, D. Mataix-Cols, A. Meyer-Lindenberg, P.K. McGuire, P. Fusar-Poli, Multimodal meta-analysis of structural and functional brain changes in first episode psychosis and the effects of antipsychotic medication, Neurosci Biobehav Rev 36(10) (2012) 2325-33. 
[13] C. Huyser, O.A. van den Heuvel, L. Wolters, E. de Haan, R. Lindauer, D.J. Veltman, A longitudinal VBM study in paediatric obsessive-compulsive disorder at 2-year follow-up after cognitive behavioural therapy, World Journal of Biological Psychiatry (2013) 1-10.

[14] A. Schienle, A. Wabnegger, W. Scharmüller, Effects of cognitive behavior therapy on regional brain volume in spider-phobic patients: preliminary results, J Anxiety Disord 28(2) (2014) 276-279.

[15] K. Månsson, P. Carlbring, A. Frick, J. Engman, C.-J. Olsson, O. Bodlund, T. Furmark, G. Andersson, Altered neural correlates of affective processing after internet-delivered cognitive behavior therapy for social anxiety disorder, Psychiatry Res 214(3) (2013) 229-237.

[16] K. Månsson, A. Frick, C.J. Boraxbekk, A.F. Marquand, S.C. Williams, P. Carlbring, G. Andersson, T. Furmark, Predicting long-term outcome of Internet-delivered cognitive behavior therapy for social anxiety disorder using fMRI and support vector machine learning, Transl Psychiatry 5 (2015) e530.

[17] A.P. Association, Diagnostic and statistical manual of mental disorders: DSM-V, 2013.

[18] G. Andersson, P. Carlbring, T. Furmark, S.O.F.I.E.R. Group, Therapist experience and knowledge acquisition in internet-delivered CBT for social anxiety disorder: a randomized controlled trial, PLoS One 7(5) (2012) e37411.

[19] G. Andersson, P. Carlbring, A. Holmström, E. Sparthan, T. Furmark, E. Nilsson-Ihrfelt, M. Buhrman, L. Ekselius, Internet-based self-help with therapist feedback and in vivo group exposure for social phobia: a randomized controlled trial, J Consult Clin Psychol 74(4) (2006) 677-686.

[20] P. Carlbring, M. Gunnarsdottir, L. Hedensjo, G. Andersson, L. Ekselius, T. Furmark, Treatment of social phobia: randomised trial of internet-delivered cognitive-behavioural therapy with telephone support, Br J Psychiatry 190 (2007) 123-8.

[21] T. Furmark, P. Carlbring, E. Hedman, A. Sonnenstein, P. Clevberger, B. Bohman, A. Eriksson, A. Hallen, M. Frykman, A. Holmstrom, E. Sparthan, M. Tillfors, E.N. Ihrfelt, M. Spak, A. Eriksson, L. Ekselius, G. Andersson, Guided and unguided self-help for social anxiety disorder: randomised controlled trial, Br J Psychiatry 195(5) (2009) 440-7.

[22] T.I. Zaider, R.G. Heimberg, D.M. Fresco, F.R. Schneier, M.R. Liebowitz, Evaluation of the Clinical Global Impression Scale among individuals with social anxiety disorder, Psychol Med 33(4) (2003) 611-622.

[23] D.M. Fresco, M.E. Coles, R.G. Heimberg, M.R. Liebowitz, S. Hami, M.B. Stein, D. Goetz, The liebowitz social anxiety scale: a comparison of the psychometric properties of self-report and clinician-administered formats, Psychol Med 31(06) (2001).

[24] K. Blair, M. Geraci, J. Devido, D. McCaffrey, G. Chen, M. Vythilingam, P. Ng, N. Hollon, M. Jones, R.J. Blair, D.S. Pine, Neural response to self- and other referential praise and criticism in generalized social phobia, Arch Gen Psychiatry 65(10) (2008) 1176-84. 
[25] J.A. Maldjian, P.J. Laurienti, R.A. Kraft, J.H. Burdette, An automated method for neuroanatomic and cytoarchitectonic atlas-based interrogation of fMRI data sets, NeuroImage 19(3) (2003) 1233-1239.

[26] P.R. Goldin, T. Manber-Ball, K. Werner, R. Heimberg, J.J. Gross, Neural mechanisms of cognitive reappraisal of negative self-beliefs in social anxiety disorder, Biol Psychiatry 66(12) (2009) 1091-9.

[27] E. Wenger, S. Kuhn, J. Verrel, J. Martensson, N.C. Bodammer, U. Lindenberger, M. Lovden, Repeated Structural Imaging Reveals Nonlinear Progression of ExperienceDependent Volume Changes in Human Motor Cortex, Cereb Cortex (2016).

[28] J. Boettcher, T. Berger, B. Renneberg, Internet-Based Attention Training for Social Anxiety: A Randomized Controlled Trial, Cogn Ther Res 36(5) (2011) 522-536.

[29] T.D. Cannon, F. Sun, S.J. McEwen, X. Papademetris, G. He, T.G. van Erp, A. Jacobson, C.E. Bearden, E. Walker, X. Hu, L. Zhou, L.J. Seidman, H.W. Thermenos, B. Cornblatt, D.M. Olvet, D. Perkins, A. Belger, K. Cadenhead, M. Tsuang, H. Mirzakhanian, J. Addington, R. Frayne, S.W. Woods, T.H. McGlashan, R.T. Constable, M. Qiu, D.H. Mathalon, P. Thompson, A.W. Toga, Reliability of neuroanatomical measurements in a multisite longitudinal study of youth at risk for psychosis, Hum Brain Mapp 35(5) (2014) 2424-34. 
Table 1. Pretreatment demographic and clinical characteristics of study subjects.

\begin{tabular}{|c|c|c|}
\hline & $\begin{array}{l}\text { Long-term CBT } \\
\text { responders } \\
(n=7)\end{array}$ & $\begin{array}{l}\text { Long-term CBT } \\
\text { non-responders } \\
(n=6)\end{array}$ \\
\hline Age, years, mean (SD) & $36.0(8.3)$ & $32.8(13.5)$ \\
\hline Age range, years & $26-47$ & $20-57$ \\
\hline Gender, female (\%) & $6(86 \%)$ & $5(83 \%)$ \\
\hline Married or de facto, $n(\%)$ & $4(57 \%)$ & $4(67 \%)$ \\
\hline \multicolumn{3}{|l|}{ Educational level, $n$ (\%) } \\
\hline Completed university & $3(43 \%)$ & $1(17 \%)$ \\
\hline Current university & $3(43 \%)$ & $0(0 \%)$ \\
\hline Lower grade & $1(14 \%)$ & $5(83 \%)$ \\
\hline Age of SAD onset, years, mean (SD) & $16.3(5.4)$ & $15.2(5.0)$ \\
\hline LSAS-SR, mean (SD) & $70.3(14.8)$ & $78.3(16.5)$ \\
\hline MADRS-S, mean (SD) & $14.4(6.9)$ & $15.7(5.6)$ \\
\hline
\end{tabular}

\footnotetext{
a Including high school, vocational school, and compulsory school

Abbreviations: CBT, Cognitive behaviour therapy; LSAS-SR, Liebowitz Social Anxiety Scale Self-report; MADRS-S, Montgomery Åsberg Depression Rating Scale - Self-report; SAD, Social anxiety disorder
} 
KNT Månsson (2016), Behavioural Brain Research

[Running title: Neuroplastic long-term effects after CBT]

Table 2. Structural GM volume, and functional BOLD treatment effects at long-term follow-up.

The ANOVA results reflect $2 \times 2$ interactions with time (pretreatment vs. 1-year follow-up) and group (responders vs. non-responders) as factors.

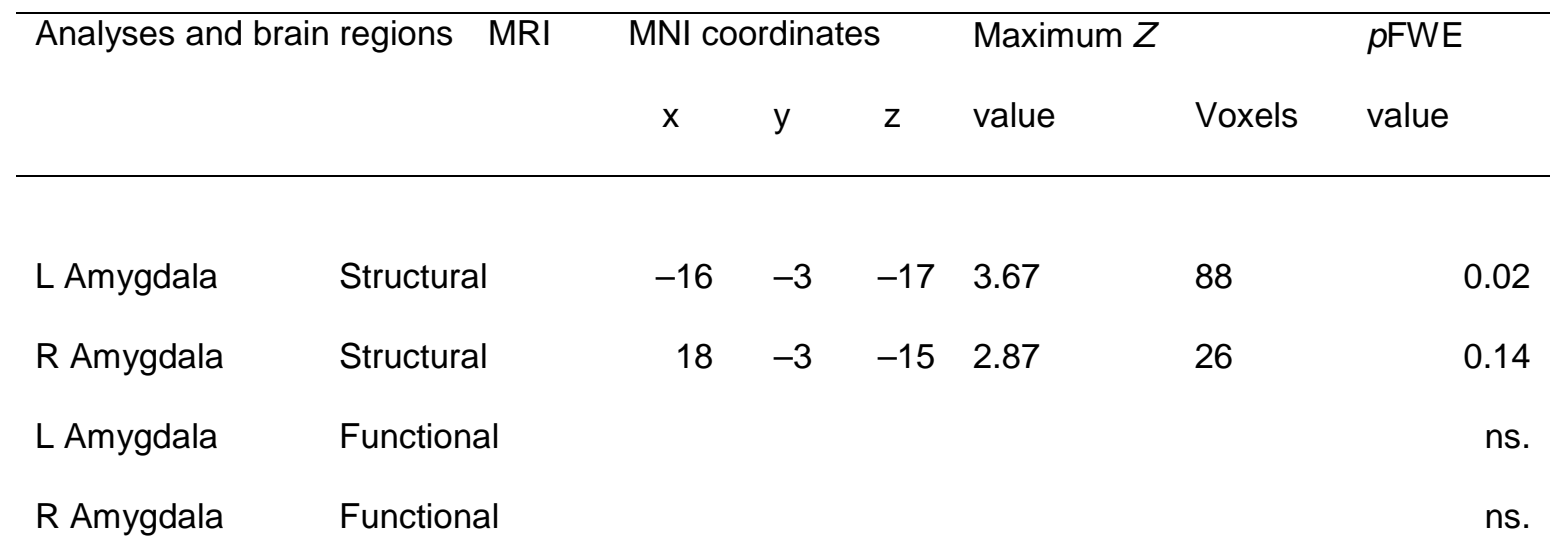

Abbreviation: BOLD, Blood-oxygen level-dependent; FWE, Family wise-error correction; GM, Gray matter; ns, Non significant 
KNT Månsson (2016), Behavioural Brain Research

[Running title: Neuroplastic long-term effects after CBT]

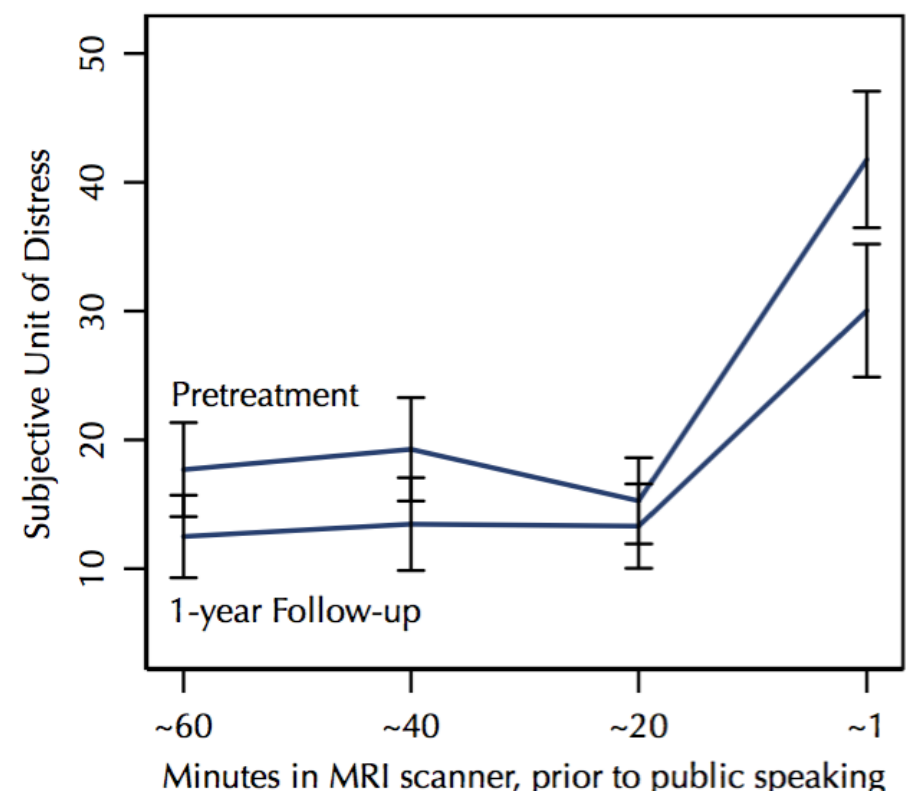

Figure 1. Subjective unit of distress were completed at 4 time-points while the subject was lying in the MRI scanner. The respective lines represent assessments at pretreatment (upper line) and 1-year follow-up (lower line). Bars indicate standard error of the mean. 
a)

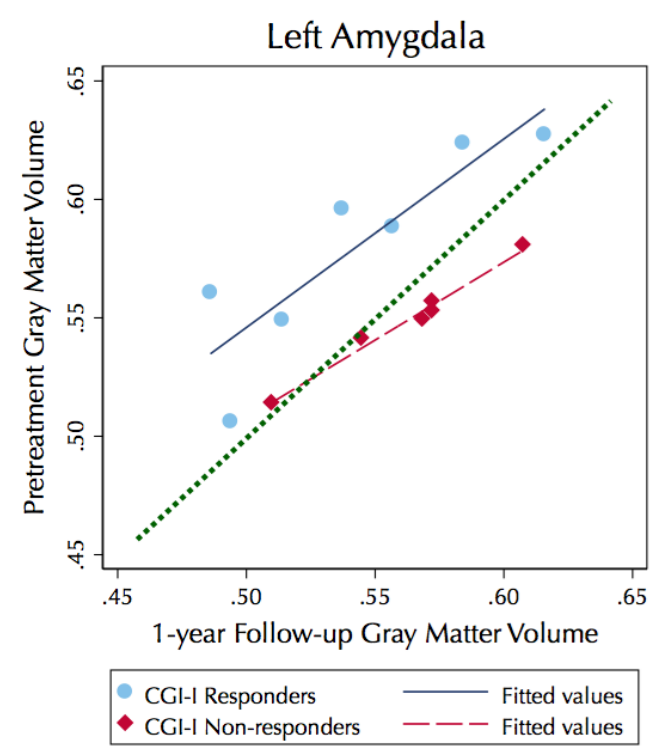

b)

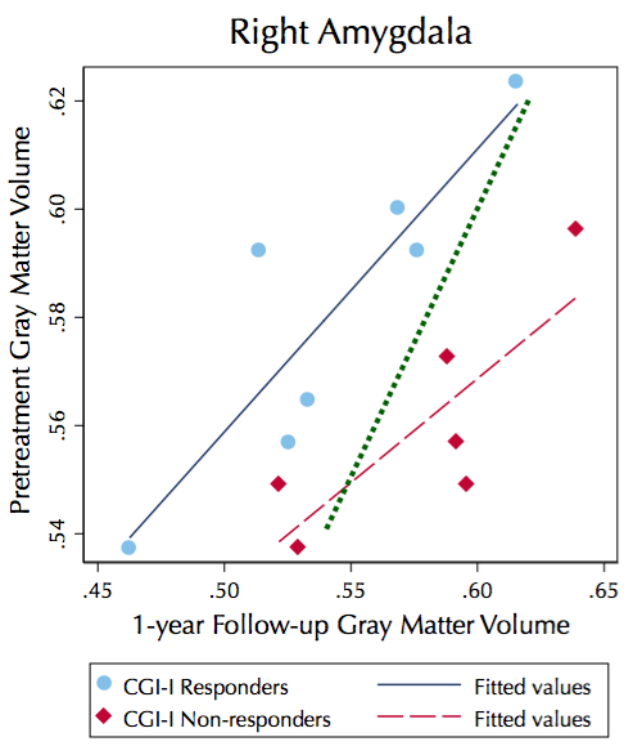

Figure 2. X-axis shows amygdala gray matter volume beta weight values in left [a], and right [b] amygdala respectively at 1-year follow-up. Y-axis demonstrates amygdala gray matter volume at pretreatment. Treatment responders (circles/solid line) and non-responders (diamonds/dashed line) are shown separately. The dotted line represents a linear non-different change from pre- to follow-up. Individuals to the left of the fitted line exhibited reduced volume, and individuals to the right of the fitted line had somewhat increased amygdala GM volume from pre-treatment to 1-year follow-up. 


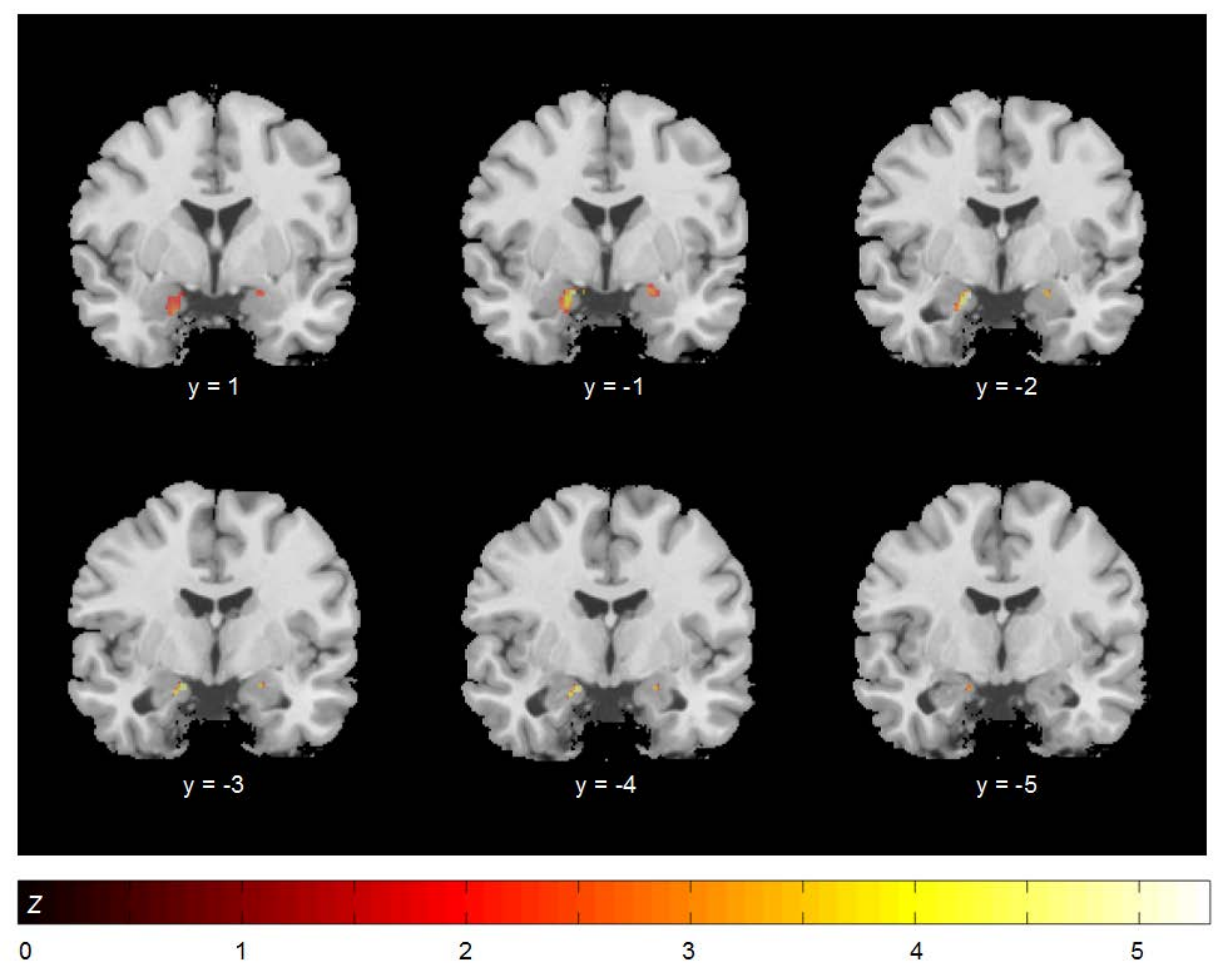

Figure 3. Gray matter volume Time $\times$ Group interaction effect in the left amygdala is displayed on multiple slices (from $y=-5$ to $y=1$ ) showing reduced volume (from pre- to 1-year follow-up) in longterm responders, relative to non-responders. Peak voxels in the left amygdala $x y z[-16,-3,-17]$ and right amygdala xyz[18, $-3,-15]$. 
KNT Månsson (2016), Behavioural Brain Research

[Running title: Neuroplastic long-term effects after CBT]

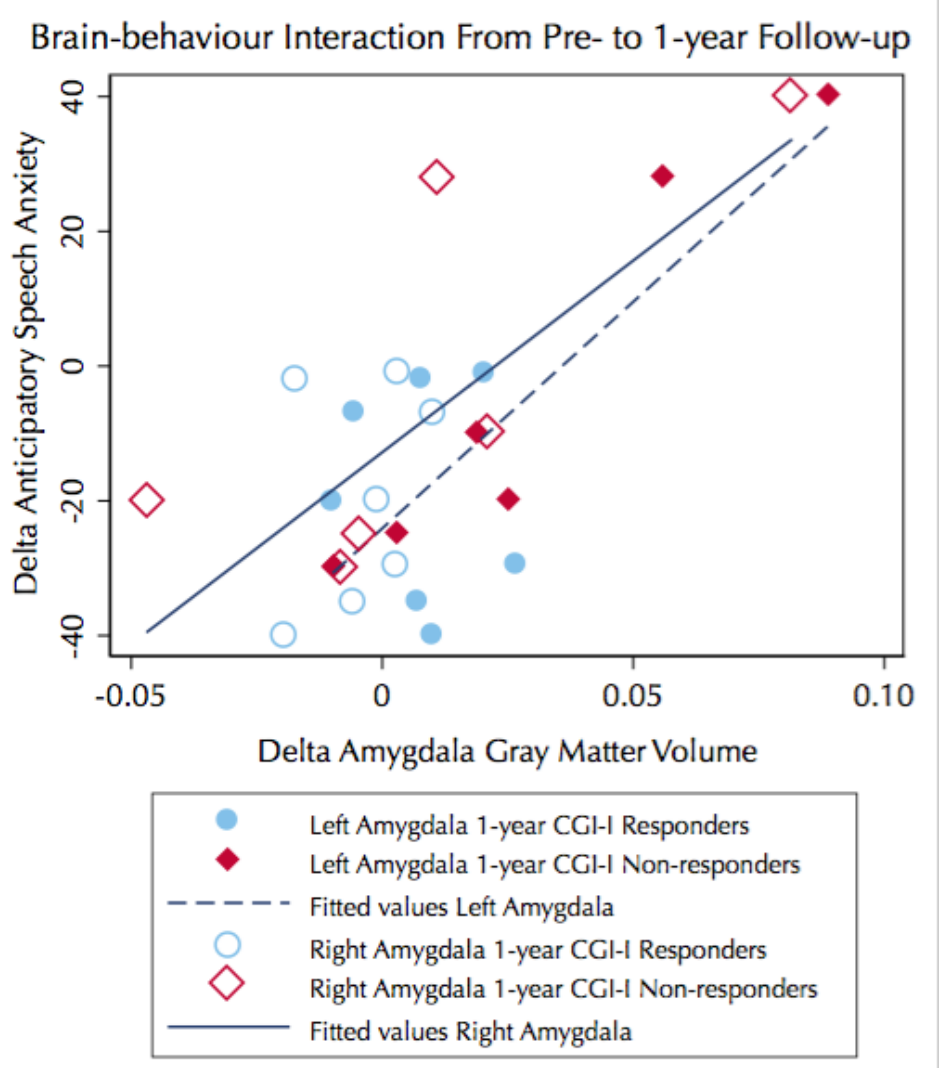

Figure 4. Altered left and right amygdala gray matter volume from pretreatment to 1 year after treatment termination was associated with improved anticipatory speech anxiety. Long-term treatment responders (circles) and non-responders (diamonds) are plotted separately. 
KNT Månsson (2016), Behavioural Brain Research

[Running title: Neuroplastic long-term effects after CBT]

a)

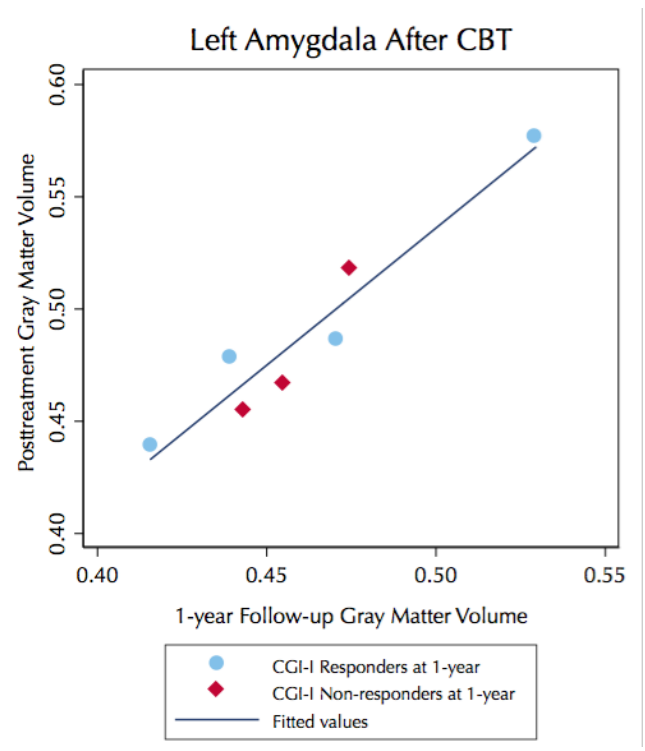

b)

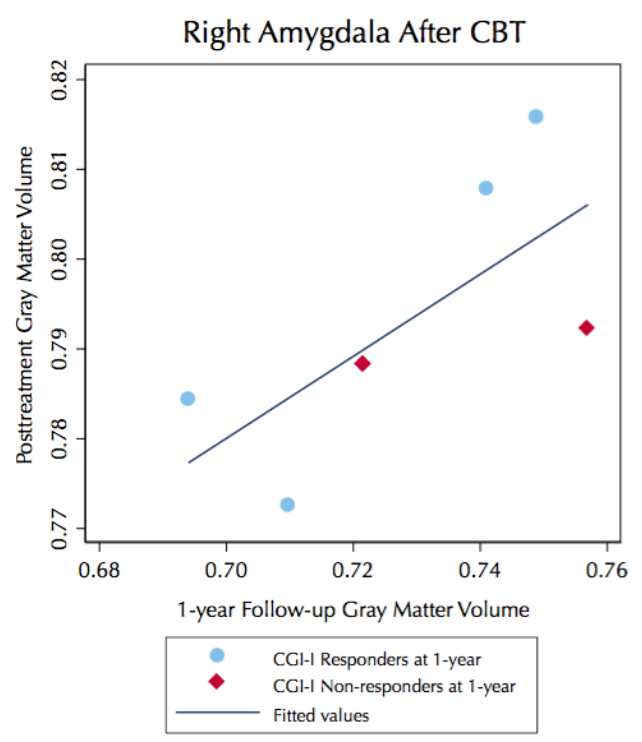

Figure 5. Long-term attenuation of amygdala gray matter volume from posttreatment ( $y$-axis) to 1-year follow-up (x-axis) after cognitive behaviour therapy (CBT), tested in 7 subjects that were initially (before the cross-over) treated with CBT. Long-term treatment responders and non-responders are plotted separately. In Figure 5b, one individual display the same peak voxel values as another individual, and is thus not shown in the figure. 